\title{
Small intestinal obstruction resulting from ischemic enteritis: a case report
}

\author{
Nobuhiro Takeuchi $\cdot$ Kazuyoshi Naba
}

Received: 7 April 2013/Accepted: 17 May 2013/Published online: 13 June 2013

(C) The Author(s) 2013. This article is published with open access at Springerlink.com

\begin{abstract}
A 69-year-old male was admitted to our institution because of a sudden onset of vomiting and abdominal distention. His past history of illness included femoral head fracture, congestive heart failure and ischaemic colitis. Plain abdominal computed tomography revealed extensively dilated small intestinal loops with a calibre change around the end of the ileum. Small intestinal obstruction was diagnosed and a transnasal ileus tube was placed. The ileus tube was constantly moved towards small intestine until it reached the distal ileum. Contrast medium from the ileus tube revealed a distal ileal stricture. Subsequently, transanal single balloon enteroscopy was performed to inspect the stricture, revealing a circumferential and afferent tubular ulcer in the distal ileum, $5 \mathrm{~cm}$ from the ileocecal valve; gastrofluorography confirmed the stricture. Although the stricture was dilated on several occasions using balloon catheters, the stricture could not be improved. However, during the treatment, his general condition worsened over time; thus, surgical treatment was decided. Operative findings revealed several circumferential ulcers with a clear margin 5-28 cm from the ileocecal valve: all lesions were successfully resected. Pathological findings were consistent with ischaemic enteritis. We report a case of small intestinal obstruction resulting from stenotic ischaemic enteritis.
\end{abstract}

Keyword Small intestinal obstruction - Ischaemic enteritis · Single balloon enteroscopy

N. Takeuchi $(\bowtie) \cdot$ K. Naba

Division of Gastroenterology, Department of Internal Medicine,

Kawasaki Hospital, 3-3-1 Higashiyama-cho, Kobe,

Hyogo 652-0042, Japan

e-mail: takeuchi_nobuhiro@kawasaki-hospital-kobe.or.jp

\section{Introduction}

Ischaemic colitis is a reversible and transient ischemic intestinal disease without occlusion of the main trunk of the mesenteric artery. Although both the large and small intestines are susceptible to ischemia, the latter rarely presents with ischaemic enteritis because of its rich collateral arteries. Because of the length of the small intestine, its inspection using endoscopy or gastrofluorography is difficult. However, with the recent advent of capsule endoscopy and balloon enteroscopy, an increasing number of small intestinal diseases have been detected. Most ischaemic enteritis cases are accompanied with hypertension, diabetes mellitus, ischaemic heart disease and cerebral infarction; thus, ischaemic enteritis is considered to be associated with atherosclerosis [1]. We report a case of small intestinal obstruction resulting from stenotic ischaemic enteritis.

\section{Case report}

A 69-year-old male was admitted to our institution because of a sudden onset of vomiting and abdominal distention in April 2012. His general history did not include smoking or alcohol consumption; however, his medical history included a femoral head fracture, which was treated with an artificial femoral head when he was 60 years old, and congestive heart failure, which improved with diuretic administration in November 2011.

His past history also included an episode of ischaemic colitis which presented with severe left-sided abdominal pain. Abdominal radiography and computed tomography revealed no abnormal findings, although colonoscopy revealed longitudinal ulcers in the descending colon 
(Fig. 1a). These findings led to a diagnosis of ischaemic colitis. Capsule endoscopy revealed circumferential ulcers in the distal small intestine (Fig. 1b) and single-balloon
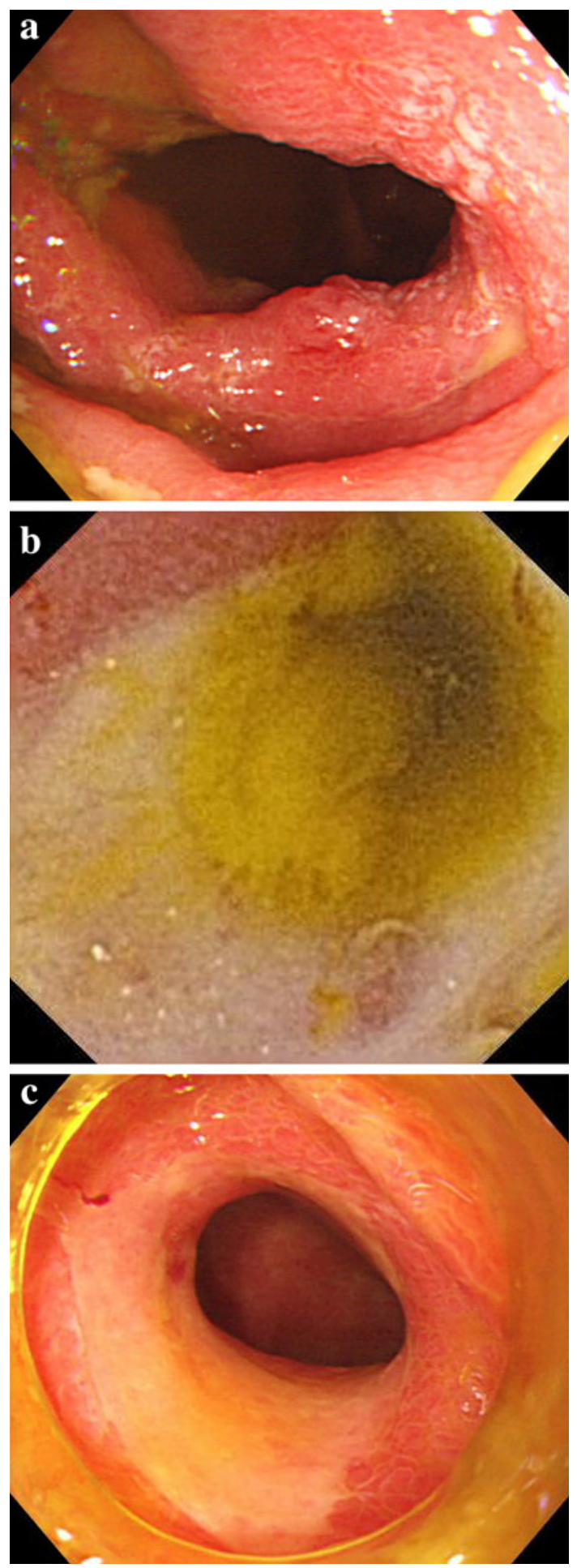

Fig. 1 Colonoscopy revealed circumferential ulceration of the descending colon (a). Capsule endoscopy revealed circumferential ulceration of the distal small intestine (b). Single-balloon enteroscopy revealed circumferential ulceration of the distal ileum (c)

enteroscopy confirmed the same in the distal ileum (Fig. 1c). Ischaemic enteritis was suspected and the patient was prescribed a treatment that included fasting and blood transfusion. Thereafter, his symptom improved rapidly.

On admission, the patient's blood pressure was $103 / 54 \mathrm{mmHg}$, heart rate was 72 beats/min, body temperature was $36.3{ }^{\circ} \mathrm{C}$ and oxygen saturation was $97 \%$ in room air. On clinical examination, his weight was $51 \mathrm{~kg}$, height was $166 \mathrm{~cm}$ and body mass index was $18.5 \mathrm{~kg} / \mathrm{m}^{2}$. Inspection of the palpebral conjunctiva revealed evidence of mild anaemia, whereas a chest auscultation revealed no abnormal findings and physical examination revealed no oedema or cyanosis. The patient's abdomen was markedly distended and reduced peristalsis was evident with mild tenderness over abdominal region. There were no palpable masses or signs of peritoneal irritation.

Blood chemistry revealed a normal white blood cell count $(7000$ cells $/ \mu \mathrm{L})$, mild anaemia (red blood cell count, $321 \times 10^{4} / \mu \mathrm{L}$; haemoglobin, $9.2 \mathrm{~g} / \mathrm{dL}$ ), elevated C-reactive protein level $(4.8 \mathrm{mg} / \mathrm{dL})$, mild hypoproteinaemia $(5.5 \mathrm{~g} / \mathrm{dL})$, mild hypoalbuminaemia $(2.4 \mathrm{~g} / \mathrm{dL})$, mildly elevated serum creatine level $(1.36 \mathrm{mg} / \mathrm{dL})$, mildly elevated blood urea nitrogen level $(25.6 \mathrm{mg} / \mathrm{dL})$, hyponatraemia $(128 \mathrm{mEq} / \mathrm{L})$ and an elevated glucose level (144 mg/ $\mathrm{dL}$; Table 1). Tuberculin and several stool culture tests

Table 1 Laboratory data on admission

\begin{tabular}{ll}
\hline Hematology & \\
WBC & $7000 / \mu \mathrm{l}$ \\
RBC & $321 \times 10^{4} / \mu \mathrm{l}$ \\
$\mathrm{Hb}$ & $9.2 \mathrm{~g} / \mathrm{dl}$ \\
$\mathrm{Ht}$ & $28.3 \%$ \\
$\mathrm{MCV}$ & $88.0 \mathrm{fl}$ \\
$\mathrm{PLT}$ & $27.2 \times 10^{4} / \mu \mathrm{l}$ \\
Biochemistry & \\
TP & $5.5 \mathrm{~g} / \mathrm{dl}$ \\
$\mathrm{Alb}$ & $2.4 \mathrm{~g} / \mathrm{dl}$ \\
$\mathrm{T}-\mathrm{Bil}$ & $1.1 \mathrm{mg} / \mathrm{dl}$ \\
$\gamma$ GTP & $179 \mathrm{IU} / \mathrm{I}$ \\
$\mathrm{ALP}$ & $434 \mathrm{IU} / \mathrm{I}$ \\
$\mathrm{AST}$ & $19 \mathrm{IU} / \mathrm{I}$ \\
$\mathrm{ALT}$ & $17 \mathrm{IU} / \mathrm{I}$ \\
$\mathrm{S}-\mathrm{Amy}$ & $58 \mathrm{IU} / \mathrm{I}$ \\
$\mathrm{LDH}$ & $168 \mathrm{IU} / \mathrm{I}$ \\
$\mathrm{BUN}$ & $25.6 \mathrm{mg} / \mathrm{dl}$ \\
$\mathrm{Cr}$ & $1.36 \mathrm{mg} / \mathrm{dl}$ \\
$\mathrm{CK}$ & $19 \mathrm{IU} / \mathrm{I}$ \\
$\mathrm{Na}$ & $128 \mathrm{mEq} / \mathrm{l}$ \\
$\mathrm{K}$ & $4.0 \mathrm{mEq} / \mathrm{l}$ \\
$\mathrm{CI}$ & $86 \mathrm{mEq} / \mathrm{l}$ \\
$\mathrm{Glu}$ & $144 \mathrm{mg} / \mathrm{dl}$ \\
$\mathrm{CRP}$ & $4.8 \mathrm{mg} / \mathrm{dl}$ \\
\hline &
\end{tabular}



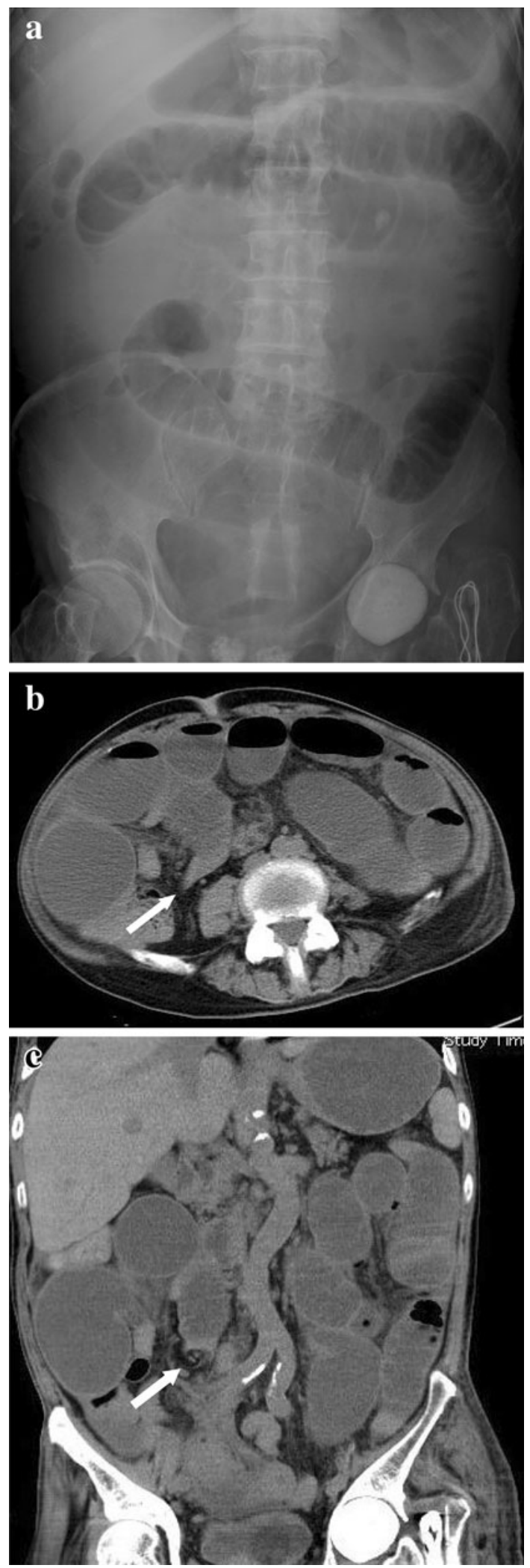

Fig. 2 Abdominal plain radiography on admission revealed dilated small intestinal loops (a). Plain abdominal computed tomography revealed extensively dilated small intestinal loops and calibre change (white arrow) around the ileal end (b axial view, $\mathbf{c}$ coronal view)
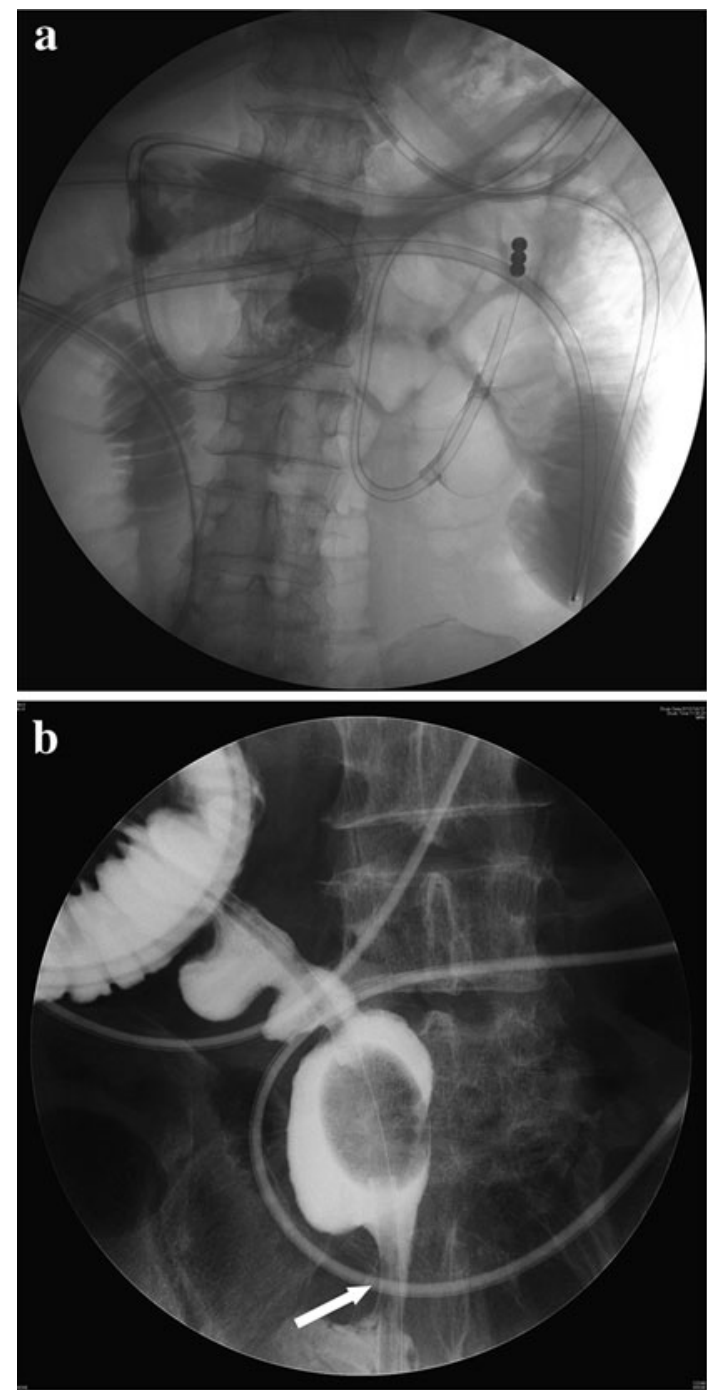

Fig. 3 A transnasal ileus tube was placed on post-admission day 1 (a). On post-admission day 4 , contrast medium from the ileus tube revealed a distal ileal stricture (white arrow) (b)

were negative. On admission, plain abdominal radiography revealed dilated small intestinal loops (Fig. 2a). Plain abdominal computed tomography revealed extensively dilated small intestinal loops, a calibre change around the ileal end and an absence of ascites (Fig. 2b, c). Therefore, small intestinal obstruction was diagnosed and a transnasal ileus tube was placed (Fig. 3a). During decompression of the small intestinal obstruction using the ileus tube, the patient was fasting and was treated with total parenteral nutrition. The ileus tube was progressively moved along the small intestine until it reached the distal ileum on postadmission day 4 . The injected contrast medium revealed a stricture in the distal ileum (Fig. 3b). Transanal singleballoon enteroscopy performed to inspect the stricture revealed a circumferential and afferent tubular ulcer in the distal ileum, $5 \mathrm{~cm}$ from the ileocecal valve (Fig. 4a); gastrofluorography confirmed the stricture (Fig. 4b), which 

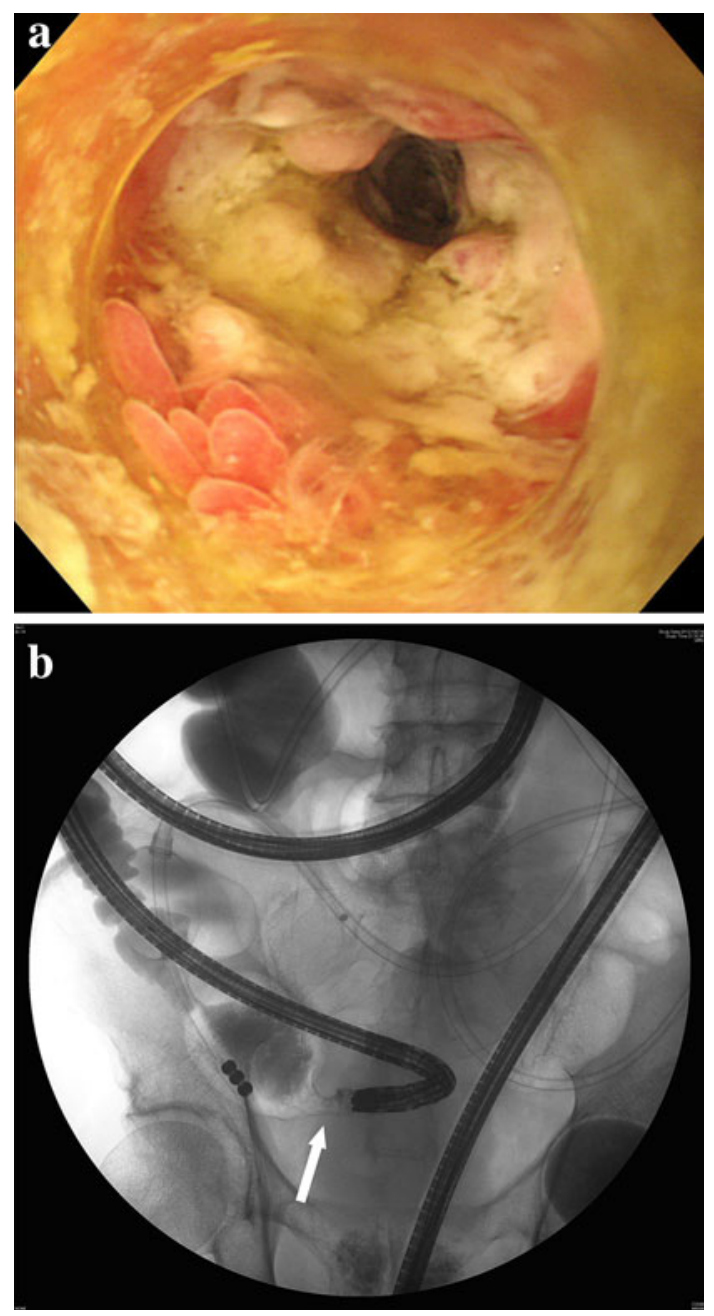

Fig. 4 Transnasal single-balloon enteroscopy revealed a circumferential and afferent tubular ulceration of the distal ileum, $5 \mathrm{~cm}$ from the ileocecal valve to the oral side (a). Gastrofluorography during the balloon enteroscopy revealed a stricture (white arrow) (b)

was dilated using balloon catheters on several occasions but could not be improved.

Despite treatment, the patient's nutritional status deteriorated and his anaemia progressed, with the latter necessitating a blood transfusion. Consequently, we decided to perform surgery on post-admission day 37 .

Operative findings revealed several strictures in the distal ileum (Fig. 5a). In total, $42 \mathrm{~cm}$ of the ileum, including the known stricture, was resected. Several circumferential ulcers with clear margins were detected in the ileum at $5-28 \mathrm{~cm}$ from the ileocecal valve (Fig. 5b). Histological findings revealed the following (Fig. 6a-c): stenotic portions of the ileum formed ulcers of grade Ul-II with an intact muscularis mucosa, the ileal lumen was covered with fibrin and fibrous connective tissue, an inflammatory cell infiltrate was present in all layers, particularly lymphocytes and eosinophils, dilatation and
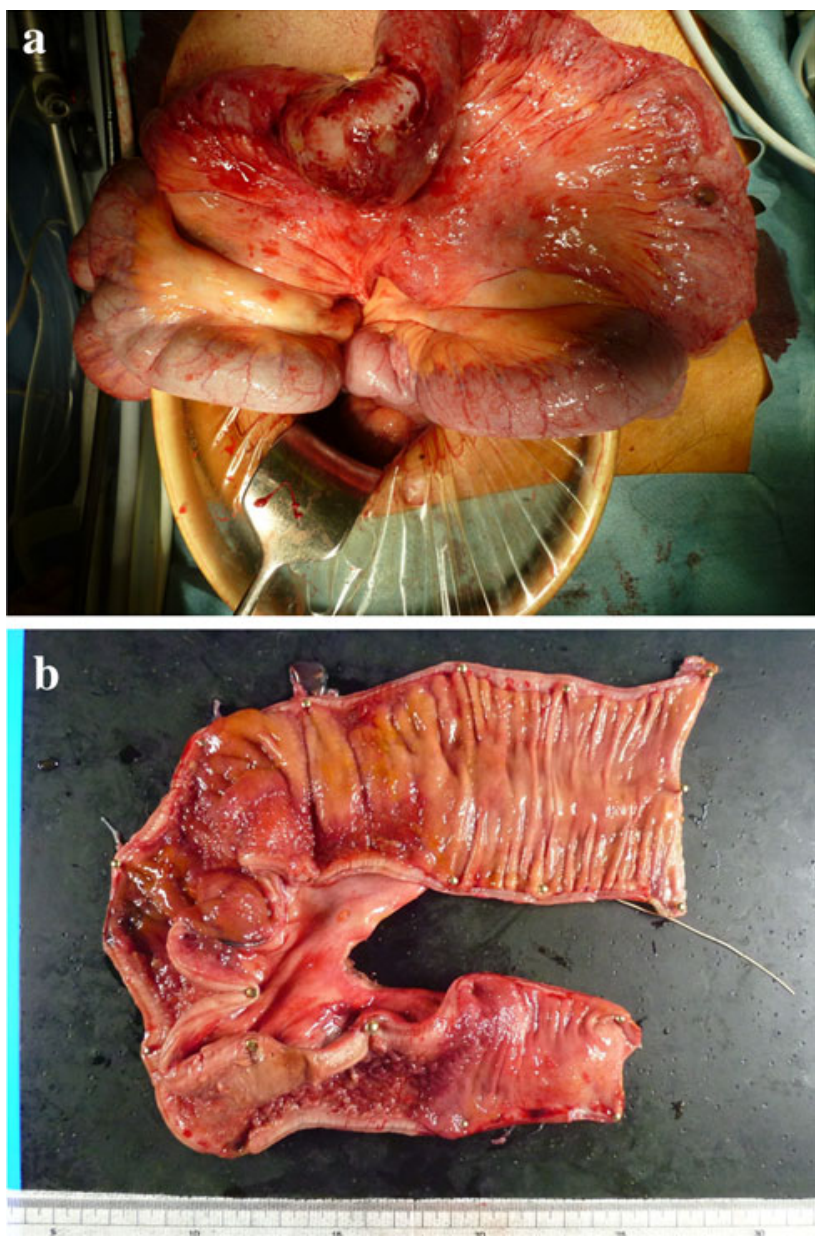

Fig. 5 Perioperative findings revealed several strictures in the distal ileum (a). In total, $42 \mathrm{~cm}$ of the ileum, including the known stricture, was resected. Several circumferential ulcers with clear margins were detected 5-28 cm from the ileocecal valve towards the oral side (b)

congestion of capillary vessels was observed in the submucosa and haemosiderin staining revealed sideroferous cells in the submucosal layers. Considering these findings, ischaemic enteritis was confirmed in the resected specimen.

The patient's post-operative course was uneventful. Oral intake was initiated and the patient experienced no recurrence of small intestinal obstruction or symptom of abdominal pain.

\section{Discussion}

Ischaemic colitis was first reported by Boley et al. [2]; and Marston et al. [3] defined it as an irreversible mucosal injury induced by intestinal ischaemia without organic occlusion of the mesenteric artery. On the basis of its severity, ischaemic colitis is classified as transient and reversible ischaemia, ischaemic ulcers with strictures and 

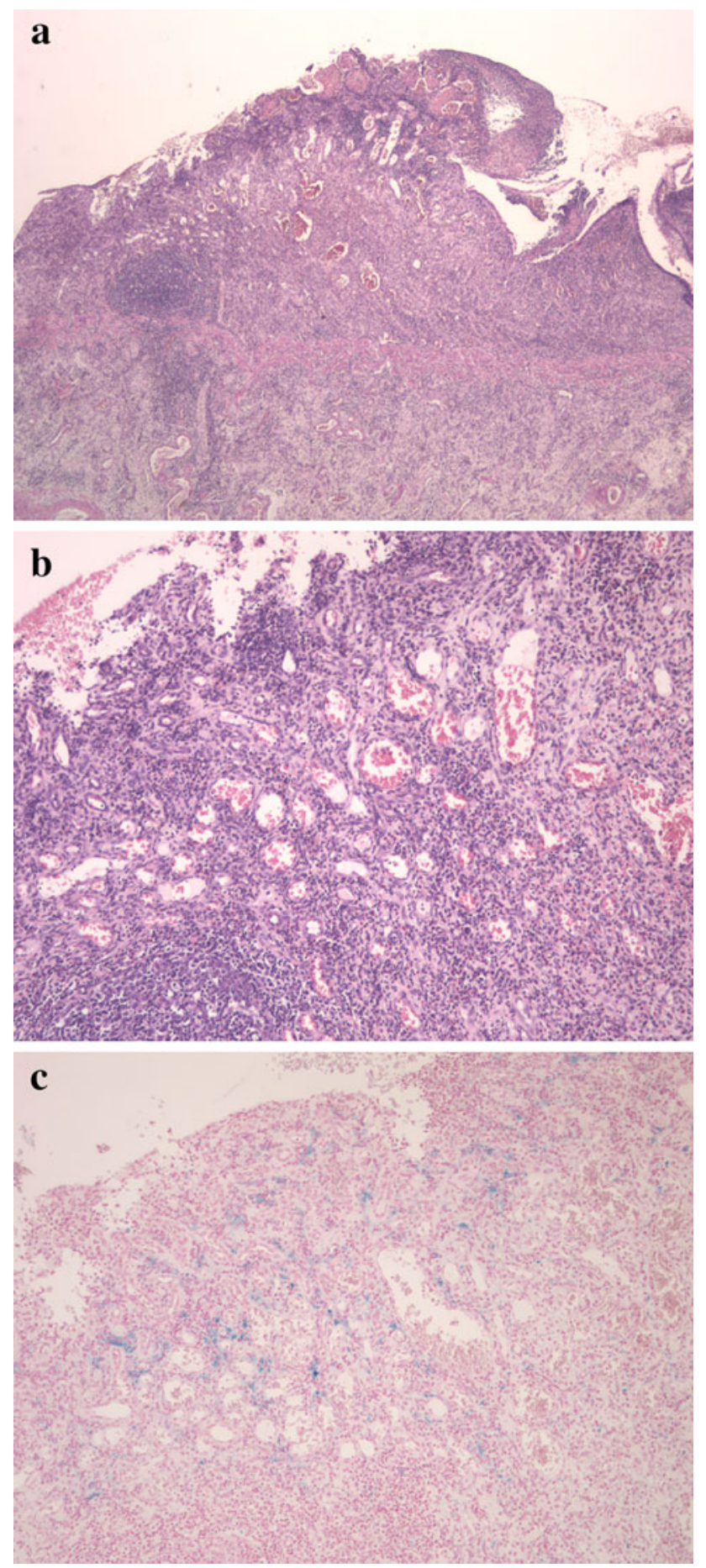

Fig. 6 Stenotic portions of the ileum formed an ulcer Ul-II excluding the muscularis mucosa. Fibrin and fibrous connective tissue covered the ileal lumen. Inflammatory cell infiltrate, particularly of lymphocytes and eosinophils, was observed throughout all layers. Dilatation and congestion of the submucosal capillary vessels were observed (H\&E staining: a low-power field, b high-power field). Haemosiderin staining revealed sideroferous cells in the submucosal layers (c)

gangrenous ischaemic colitis. Subsequently, gangrenous ischaemic colitis was removed from the classification because the progress of intestinal necrosis is irreversible at this stage. In the small intestine, the same condition as ischaemic colitis is termed ischaemic enteritis, which is fairly rare and shares several similarities with ischaemic colitis. Both ischaemic colitis and enteritis are considered to be caused by hypoperfusion of the mesenteric vessels.

There are several factors accounting for the rarity of ischaemic enteritis. First, the small intestine rarely presents with ischaemic changes because of its rich collateral flow [4]. Second, there are no established clinical or pathological diagnostic criteria for ischaemic enteritis. Third, the small intestine is not easy to examine; therefore, although small bowel pathology is rare, examination of acute small intestinal disease is liable to be delayed and its detection unsuccessful [5].

Patients with ischaemic enteritis commonly present with abdominal pain, nausea, fever and vomiting, while melena is rare. Stenotic ischaemic enteritis occasionally presents with small intestinal obstruction after resolution of the initial symptoms. Ischaemic enteritis is classified as transient type, when it resolves completely within a few days without sequelae, or as stenotic type, when it presents with tubular stricture after the course of chronic inflammation $[6,7]$. Some case reports $[8,9]$ demonstrated a small bowel obstruction due to multiple ulcerations of ischaemic enteritis with circumferential strictures which required surgical resection. Before the advent of capsule endoscopy and balloon enteroscopy, it was difficult to observe small intestinal lesions; therefore, most transient ischaemic enteritis cases could have been missed. The difference between transient and stenotic types may depend on the severity of ischaemia.

Ischaemic enteritis is characterized by the formation of strictures, circumferential segmental ulcers and afferent tubular stenosis, but seldom presents with longitudinal ulcers. In contrast, ischaemic colitis is characterized by longitudinal ulcers with eccentric deformation and sacculation. Strictures in ischaemic colitis tend to spontaneously resolve over time, whereas those in ischaemic enteritis sometimes tend to progressively worsen and ultimately result in total occlusion [10]. Therefore, because the strictures of ischaemic enteritis may fail to resolve even after prolonged periods of conservative management, surgical intervention should be considered sooner rather than later. In our case, distal ileal lesions formed a stricture with afferent tubular ulcers. The stricture on which balloon dilatation was performed was refractory to several attempts at balloon dilatation; therefore, the stricture was surgically resected.

The macroscopic characteristics of ischaemic enteritis are: afferent tubular stenosis, circumferential stenosis with clear boundaries and severe thickening of the intestinal wall. The histological characteristics of ischaemic enteritis include the following $[6,9]$ : 
1. Variable ulcer depth with most at Ul-II or Ul-III

2. Ulcer bases lined with vessel-rich granulation tissue

3. Severe fibrosis seen mainly within the submucosal layers

4. Severe inflammatory cell invasion (primarily lymphocytes and plasma cells)

5. Haemosiderin-laden macrophages scattered throughout the entire thickness of the intestine.

Our case was consistent with ischaemic enteritis both macroscopically and microscopically. Endoscopic analysis revealed circumferential, afferent, tubular and segmental ulcers with clear boundaries, whereas microscopic analysis revealed inflammatory cell infiltration throughout all layers, with dilatation and congestion of submucosal capillary vessels. Moreover, haemosiderin staining revealed the presence of sideroferous cells in the submucosa.

The differential diagnoses in our case included small bowel tuberculosis, Crohn's disease and non-specific multiple ulcers of the small intestine. These diagnoses were excluded because of the absence of cryptogenic abscesses and non-caseating granulomas.

\section{Conclusions}

We reported a rare case of small intestinal obstruction resulting from stenotic ischaemic enteritis. When encountering small intestinal ulcerated lesions with tubular afferent stenosis, a diagnosis of ischaemic enteritis should be considered. Ischaemic enteritis may result in small intestinal obstruction due to intestinal stenosis in its chronic phase. Therefore, when a diagnosis of ischaemic enteritis is confirmed using enteroscopy or gastrofluorography, surgical treatment should be the intervention of choice.
Conflict of interest The authors declare that they have no conflict of interest.

Open Access This article is distributed under the terms of the Creative Commons Attribution License which permits any use, distribution, and reproduction in any medium, provided the original author(s) and the source are credited.

\section{References}

1. Friedman G, Sloan WC. Ischaemic enteropathy. Surg Clin N Am. 1972;52:1001-12.

2. Boley SJ, Schwartz S, Lash J, Sternhill V. Reversible vascular occlusion of the colon. Surg Gynecol Obstet. 1963;116:53-60.

3. Marston A, Pheils MT, Thomas ML, Morson BC. Ischaemic colitis. Gut. 1966;7:1-15.

4. Robinson JW, Mirkovitch V, Winistörfer B, Saegesser F. Response of the intestinal mucosa to ischaemia. Gut. 1981;22:512-27.

5. Sidhu R, Sanders DS, Morris AJ, McAlindon ME. Guidelines on small bowel enteroscopy and capsule endoscopy in adults. Gut. 2008;57:125-36.

6. Iida M, Matsui T, Yao T, Iwashita A, Sakamoto K, Fuchigami T, Yao T, Fujishima M. Radiographic features in ischemic jejunoileitis: serial changes and comparison with pathologic findings. Gastrointest Radiol. 1992;17:327-32.

7. Yu J, Kim B, Chung S, Park CW, Chang YS. Ischaemic enteritis in a patient with chronic renal failure: diagnosis and management decisions. BMJ Case Rep. 2010. pii: bcr0920092249.

8. Kato T, Morita T, Fujita M, Miyasaka Y, Senmaru N, Hiraoka K, Horita $\mathrm{S}$, Kondo $\mathrm{S}$, Kato $\mathrm{H}$. Ischemic stricture of the small intestine associated with acute pancreatitis. Int $\mathrm{J}$ Pancreatol. 1998;24:237-42.

9. Todani T, Sato Y, Watanabe Y, Toki A, Uemura S. Ischemic jejunal stricture developing after diabetic coma in a girl: a case report. Eur J Pediatr Surg. 1993;3:115-7.

10. Yang XY, Chen CX, Zhang BL, Yang LP, Su HJ, Teng LS, Li YM. Diagnostic effect of capsule endoscopy in 31 cases of subacute small bowel obstruction. World $\mathrm{J}$ Gastroenterol. 2009;15:2401-5. 